\title{
TACROLIMO DE LIBERAÇÃO MODIFICADA
}

\author{
Modified-release Tacrolimus
}

\author{
Claudia Rosso Felipe, Jose Osmar Medina Pestana, Helio Tedesco Silva Jr.
}

\section{RESUMO}

Tacrolimo de liberação modificada (TAC-MR) é uma nova formulação de (?) que permite a administração de uma dose única diária. Em voluntários sadios e em receptores de transplante de rim e fígado, a exposição ao tacrolimo (ASC - área sob a curva da concentração durante o intervalo de dosagem) e as concentrações residuais foram semelhantes após a administração de TAC ou TAC-MR. A conversão de TAC para TAC-MR (1:1 mg) é eficaz e segura, utilizando a mesma estratégia de monitoramento e a mesma faixa terapêutica. A equivalência terapêutica entre TAC-MR e TAC foi comprovada em um estudo prospectivo, multicêntrico, randomizado e aberto que incluiu 638 novos receptores de transplante renal. As médias das doses e das concentrações sanguíneas residuais de tacrolimo, a influência da etnia e a dificuldade para atingir a faixa terapêutica foram semelhantes entres os receptores de transplante renal que receberam TAC ou TAC-MR. Também não foram observadas diferenças na proporção de pacientes que apresentou falha de eficácia (desfecho composto por rejeição aguda comprovada por biópsia, perda do enxerto, óbito ou perda de seguimento) e no perfil de segurança. Os resultados desses estudos indicam que TAC-MR, administrado em dose única diária apresenta eficácia e segurança semelhante à do TAC, permitindo a utilização da mesma estratégia de monitoramento terapêutico. Estudos de seguimento prolongado são necessários para avaliar a influência de TACMR na adesão do paciente ao tratamento, bem como se as diferenças farmacocinéticas podem resultar em redução significativa de reações adversas, fatores com reconhecido impacto na sobrevida do paciente transplantado.

Descritores: Tacrolimo, Transplante renal, Imunossupressão.

\section{Instituição:}

Hospital do Rim e Hipertensão - Disciplina de Nefrologia, Universidade Federal de São Paulo, São Paulo, SP, Brazil.

\section{Correspondência:}

Helio Tedesco-Silva Junior, MD.

Rua Borges Lagoa, 960, Vila Clementino, São Paulo - SP - Brazil - CEP 04038-002

Tel: (11) 5087-8056 Fax: (11) 5087-8008.

E-mail: heliotedesco@hrim.com.br

Conflito de interesse: Helio Tedesco-Silva Junior recebeu financiamento da JanssenCilag Farmacêutica.

Recebido em: 11.12 .2007

Aceito em: 10.01.2008

\section{INTRODUÇÃO}

A ampla utilização clínica de novas drogas imunossupressoras nas últimas duas décadas foi um dos fatores determinantes da redução progressiva na incidência de rejeição aguda e do aumento significativo na sobrevida do enxerto no primeiro ano após o transplante de órgãos vascularizados. ${ }^{1}$

Tacrolimo (TAC) foi aprovado nos Estados Unidos para profilaxia da rejeição aguda após transplante hepático (1994) e renal (1997), e no Brasil obteve seu registro junto ao Ministério da Saúde em 1999. TAC é uma droga imunossupressora com eficácia amplamente comprovada. Em uma recente meta-análise dos principais estudos clínicos randomizados, TAC mostrou eficácia superior na prevenção da rejeição aguda (39\% de redução no risco relativo de rejeição aguda comprovada por biópsia) e da perda do enxerto $(10 \%$ de redução no risco relativo de perda do enxerto, incluindo óbito com rim funcionante), ao final do primeiro ano do transplante, quando comparado com a ciclosporina. O risco de rejeição aguda resistente ao tratamento com esteroides também é menor em pacientes recebendo TAC comparado com aqueles recebendo ciclosporina ( $51 \%$ de redução no risco relativo). Esses achados são independentes do uso de indução com preparação de anticorpos mono ou policlonais e do tipo de droga imunossupressora coadjuvante - azatioprina, micofenolato mofetil, sirolimo. ${ }^{2} \mathrm{~A}$ incidência de rejeição aguda sub-clínica, fator de risco para deterioração progressiva da função renal e para a perda precoce do 
enxerto também é menor em pacientes em uso de TAC comparado com a ciclosporina. ${ }^{3}$ Por outro lado, TAC está associado a uma maior incidência de diabete melito dependente de insulina (risco relativo $70 \%$ maior comparado com a ciclosporina) e reações adversas neurológicas e gastrintestinais após o transplante. Finalmente, o uso de TAC está associado ao melhor controle da pressão arterial, menor concentração de colesterol e melhor função renal (1.4 versus $1.7, \mathrm{p}=0.0014)$ em longo prazo comparado com a ciclosporina. ${ }^{2,4}$

TAC é administrado em duas doses diárias iguais e requer monitoramento rotineiro da sua concentração no sangue (concentração residual medida imediatamente antes da administração da dose matinal) para reduzir o risco de rejeição aguda - associado a concentrações reduzidas - e o risco de reações adversas associado a concentrações elevadas. Entretanto, a absorção do TAC é significativamente menor no período noturno, ${ }^{5}$ sugerindo que a administração de uma dose única diária poderia ser clinicamente eficaz, desde que alcançasse concentrações sanguíneas residuais semelhantes àquelas observadas com duas doses diárias. Uma vez que a falta de adesão ao tratamento está associada a um maior risco de perda do enxerto, essa facilidade posológica poderia reduzir esse risco, e possivelmente prolongar a sobrevida do enxerto.

Através de estudos farmacêuticos em voluntários sadios utilizando quatro formulações modificadas de TAC (MR-1, MR-2, MR-3 e MR-4) foi observado que a administração de uma dose única diária da formulação MR4 produzia uma absorção similar àquela observada com TAC em duas tomadas diárias. Essa característica farmacocinética, bem como a eficácia e segurança clínicas foram confirmadas posteriormente em estudos com receptores de transplante renal e hepático. ${ }^{6}$ TAC-MR (tacrolimo de liberação modificada, MR4) é uma nova formulação de tacrolimo de liberação modificada, que permite a administração de uma dose única diária produzindo concentrações residuais terapêuticas equivalentes à formulação original (Figura 1).

Figura 1. Curva da concentração sanguínea de tacrolimo em receptores de transplante renal após a administração de TAC (pontos preenchidos) ou TAC-MR (pontos abertos).

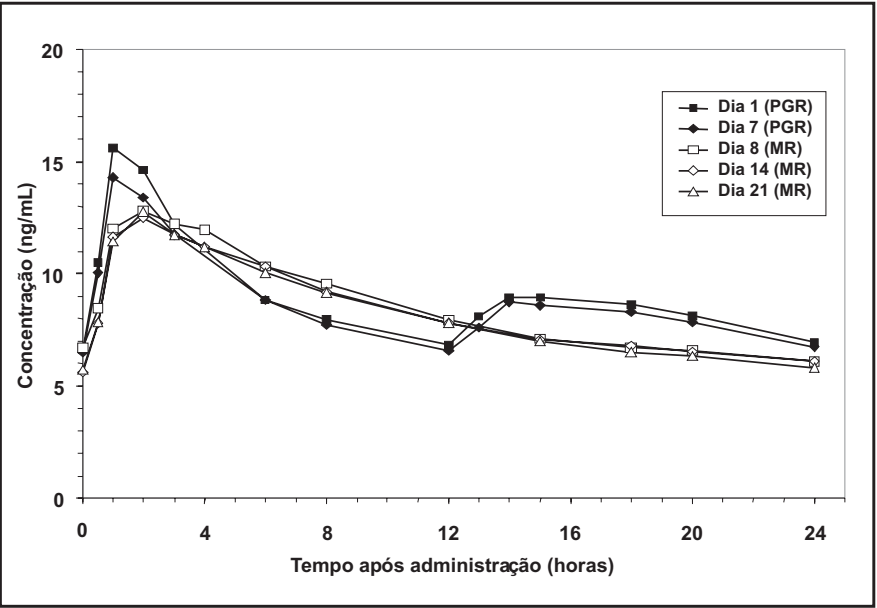

\footnotetext{
* A nomenclatura MR (Modified Release) foi utilizada nos estudos clínicos referidos nesta revisão, mas no Brasil esta medicação está registrada como Tacrolimo, cápsulas de liberação prolongada.
}

\section{MECANISMOS DE AÇÃO E FARMACODINÂMICA}

TAC é um imunossupressor macrolídeo que inibe tanto a resposta imune celular quanto a humoral através de vários mecanismos de ação. TAC forma um complexo molecular com a imunofilina FKBP12 (FK506 binding protein 12) bloqueando a atividade da enzima calcineurina, uma serina-treonina fosfatase. Essa inibição previne a migração nuclear de fatores de transcrição citoplasmáticos que promovem a ativação de genes relacionados à ativação da célula T. TAC inibe a produção de citocinas envolvidas no processo de rejeição aguda mediada por células assim como citocinas envolvidas na estimulação de linfócitos B e na produção de anticorpos.?

A potência relativa do TAC na inibição da resposta imune é 10 a 100 vezes maior que a observada com ciclosporina. Apesar dessa maior potência farmacodinâmica, não há necessariamente uma variação do número ou da gravidade das reações adversas. Da mesma forma que a ciclosporina, TAC pode causar nefrotoxicidade através de vários mecanismos, incluindo a ativação do fator nuclear $\kappa \beta$, aumento dos níveis de endotelina e tromboxane A2 e aumento da expressão de genes associados a fibrose, tais como TGF- $\beta$, colágeno e metaloproteinase proteolítica da matriz. O efeito diabetogênico do TAC, caracterizado pela redução da sensibilidade à insulina e das reservas insulínicas das células $\beta$ e as reações adversas neurológicas possivelmente relacionadas ao aumento de apoptose nas células endoteliais dos capilares do cérebro são maiores que os observados em pacientes em uso de ciclosporina. Em contrapartida, TAC apresenta menor efeito na concentração plasmática de colesterol, quando comparado com a ciclosporina.,

\section{ESTUDOS EM VOLUNTÁRIOS SADIOS}

Em voluntários sadios, TAC-MR foi bem tolerado e apresentou um perfil de segurança similar ao de TAC. Nos estudos de dose única ou doses repetidas, não se observou diferença nas concentrações de TAC (ASC: área sob a curva da concentração de TAC durante o intervalo de dosagem). No estado de equilíbrio, a razão das médias geométricas das concentrações de tacrolimo após a administração de TAC-MR ou de TAC $\left[\mathrm{ASC}_{(0-24 \mathrm{~h})} /\left(\mathrm{ASCdia}_{(0-12 \mathrm{~h})}+\mathrm{ASCnoite}_{(0-12 \mathrm{~h})}\right)\right]$ atingiu o critério de bioequivalência (80-125\%) recomendado pelo FDA (Food and Drug Administration), apesar da concentração máxima de tacrolimo $\left(\mathrm{C}_{\max }\right)$ ter sido menor após administração de TAC-MR (Figura 1). As concentrações residuais mínimas $\left(\mathrm{C}_{\mathrm{min}}\right)$ de tacrolimo após a administração de TAC-MR e TAC foram similares nos estudos de doses repetidas (Tabela 1). Além disso, a correlação entre Cmin e ASC foi numericamente maior após a administração de TAC-MR, sugerindo a possibilidade da sua utilização empregando a mesma faixa terapêutica rotineiramente aplicada em receptores de transplante renal e hepático em uso de TAC. ${ }^{1}$

\section{CONVERSÃO DE TAC PARA TAC-MR EM RECEPTORES ESTÁVEIS DE TRANSPLANTE RENAL E HEPÁTICO}

Em transplantados renais estáveis $(n=70)$ a conversão de TAC para TAC-MR, utilizando doses diárias totais equivalentes (1:1) foi bem tolerada; o perfil de eventos adversos não foi diferente e as concentrações obtidas foram similares. No estado de equilíbrio, a razão entre as médias geométricas das concentrações (ASC) de tacrolimo após a administração de TAC-MR e TAC foi de $94.7 \%$ (Tabela 1). As médias dos valores de Cmin não foram diferentes, observando-se elevada correlação dessas com a ASC, após a 
Tabela 1. Comparação de parâmetros farmacocinéticos de tacrolimo (ASC e Cmin) em receptores de transplante renal e hepático.

\begin{tabular}{|c|c|c|c|c|}
\hline Estudo & Parâmetros & TAC & TAC-MR & Razão (90\% IC) \\
\hline \multirow{4}{*}{ Transplantados renais estáveis } & ASC (ng,h/mL) & $206,6 \pm 58,4$ & $197,6 \pm 47,5$ & \multirow{4}{*}{$\begin{array}{l}94,97 \\
(90,72-99,41)\end{array}$} \\
\hline & Cmin (ng/mL) & $6,73 \pm 1,99$ & $5,83 \pm 1,63$ & \\
\hline & $\mathrm{R}$ & $>0,80$ & $>0,86$ & \\
\hline & Dose (mg) & $5,8 \pm 3,5$ & $6,1 \pm 3,5$ & \\
\hline \multirow{4}{*}{ Transplantados hepáticos estáveis } & AUC (ng,h/mL) & $215,6 \pm 77,8$ & $187,9 \pm 58,1$ & \multirow{4}{*}{$\begin{array}{l}88,79 \\
(85,42-92,29)\end{array}$} \\
\hline & Cmin (ng/mL) & $7,1 \pm 2,5$ & $5,8 \pm 1,8$ & \\
\hline & $\mathrm{R}$ & $>0,86$ & $>0,88$ & \\
\hline & Dose (mg) & $5,2 \pm 3,48$ & $5,3 \pm 3,36$ & \\
\hline \multirow{4}{*}{ Transplantados hepáticos pediátricos } & $\operatorname{AUC}(\mathrm{ng}, \mathrm{h} / \mathrm{mL})$ & 179,58 & 181,22 & \multirow{4}{*}{$\begin{array}{l}100,9 \\
(90,8-112,1)\end{array}$} \\
\hline & Cmin (ng/mL) & 5,35 & 4,91 & \\
\hline & $\mathrm{R}$ & 0,94 & 0,90 & \\
\hline & Dose (mg) & $5,3 \pm 3,27$ & $5,4 \pm 3,40$ & \\
\hline
\end{tabular}

administração de TAC ( $>0.80)$ ou de TAC-MR (r>0.86). Entre pacientes de etnia negra, a variabilidade intra-individual nas concentrações de tacrolimo foi até quatro vezes menor após o uso de TAC-MR comparado com TAC (relação TAC-MR/TAC $=0,235, \mathrm{p}=0,024),{ }^{9}, 10$ Resultados semelhantes foram observados em receptores de transplante hepático adulto ${ }^{11}$ e pediátrico ${ }^{12}$ após conversão de TAC a TAC-MR (Tabela 1).

Após dois anos de seguimento no estudo em transplantados renais, 59 receptores estavam estáveis. Quatro pacientes desenvolveram episódios de rejeição aguda comprovada por biópsia. Oito pacientes interromperam o uso de TAC-MR devido à ocorrência de um evento adverso; perdeu-se o seguimento de um paciente e um paciente perdeu a função do enxerto devido a nefropatia por polioma vírus, retornado ao tratamento dialítico. ${ }^{13}$ No estudo em transplantados hepáticos, 57 receptores estavam estáveis após dois anos. Quatro pacientes desenvolveram episódios de rejeição aguda comprovada por biópsia. Três desenvolveram diabete melito após o transplante, oito interromperam o uso de TAC-MR devido à ocorrência de um evento adverso, cinco retiraram o consentimento informado para o estudo e um paciente faleceu como conseqüência de uma neoplasia pulmonar. ${ }^{14}$ Entre os receptores pediátricos de transplante hepático não foi observado qualquer episódio de rejeição aguda, perda do enxerto ou óbito. O tratamento com TAC-MR não foi descontinuado em nenhum receptor e não houve ocorrência de diabete melito. ${ }^{15}$

Esses estudos demonstraram a eficácia e segurança da conversão de TAC para TAC-MR em receptores estáveis de transplante renal e hepático. A biodisponibilidade (ASC) do tacrolimo e a faixa terapêutica da concentração sanguínea residual (Cmin) observadas após a conversão para TAC-MR (1:1) foram equivalentes àquelas observadas durante a administração de TAC TAC, sem a necessidade de alteração significativa na dose de TAC-MR. A correlação entre a exposição (AUC0-24) e a Cmin observada após a conversão para TAC-MR é adequada para o monitoramento terapêutico após o transplante.

\section{ESTUDOS EM RECEPTORES DE TRANSPLANTE RENAL DE NOVO}

Em um estudo piloto randomizado, aberto e multicêntrico que incluiu 66 receptores de transplante renal (TAC, $n=32$ vs. TACMR, $n=34$ ), a incidência de rejeição aguda foi de $15 \%$ e $13 \%$, respectivamente. Nesse estudo, os valores da ASC de TACMR e TAC foram comparáveis em estado de equilíbrio (dia 14 e semana 6). Houve boa correlação entre ASC e Cmin, tanto para TAC-MR quanto para TAC (aproximadamente $\mathrm{r}=0.9$ para ambas formulações).$^{16}$ Resultados semelhantes foram obtidos em um estudo com desenho semelhante, envolvendo receptores de transplante hepático (TAC, $\mathrm{n}=32$ vs. TAC-MR, $\mathrm{n}=45$ ). A incidência de rejeição aguda foi de $27 \%$ nos dois grupos. ${ }^{17}$ Nesses dois estudos não se observou diferença no perfil de segurança comparando as duas formulações de TAC.

A equivalência terapêutica (similar incidência de rejeição aguda e de reações adversas) do TAC-MR comparada com TAC observada nesses estudos pilotos permitiram a realização do primeiro estudo de fase III, prospectivo, multicêntrico, randomizado e aberto, que incluiu 638 novos receptores de transplante renal seguidos por um ano. Todos os receptores receberam indução com basiliximab, micofenolato mofetil ( $2 \mathrm{~g} / \mathrm{dia})$ e prednisona. Os 214 receptores do grupo controle receberam ciclosporina microemulsão (Neoral®) enquanto que 214 receptores TAC e outros 219 receberam TACMR. Aproximadamente 50\% eram receptores de transplantes de doador falecido, $20 \%$ de etnia negra, $26 \%$ portadores de diabete melito e $19 \%$ apresentaram função retardada do enxerto. As doses médias de TAC ou TAC-MR necessárias para atingir a faixa terapêtica de tacrolimo proposta pelo estudo foram semelhantes. Não foram observadas diferenças estatísticas nas médias das concentrações residuais de tacrolimo nos dois grupos (Figura 2A). Também não foram observadas diferenças na proporção de pacientes que atingiram as faixas terapêuticas de tacrolimo proposta pelo estudo, confirmando que TAC-MR não 
Figura 2. A) Médias das concentrações residuais de tacrolimo no dia 3 (D3) e meses 2 (M2) e 4 (M4) em receptores de transplante renal que receberam TAC ou TAC-MR. B) Proporção de pacientes (\%) recebendo TAC e TAC-MR que não atingiu a faixa de concentração sanguínea de tacrolimo preconizada pelo estudo.

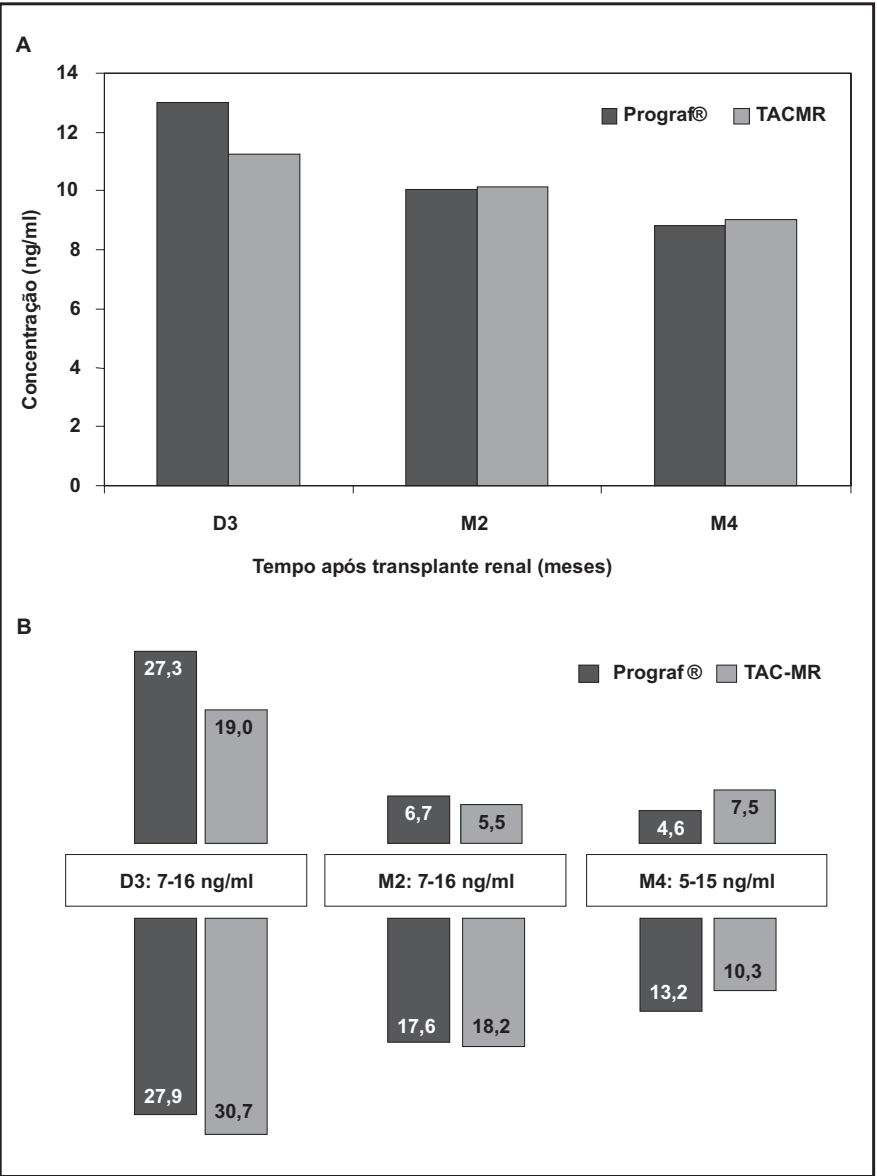

oferece maior dificuldade para implementação do monitoramento terapêutico rotineiro (Figura $2 \mathrm{~B}$ ). Como já reportado para TAC, pacientes de etnia negra também necessitaram de doses maiores de TAC-MR para atingir concentrações sanguíneas comparáveis às observadas em pacientes brancos.

Ao final de um ano, a proporção de pacientes que apresentou falha de eficácia (desfecho composto por rejeição aguda comprovada por biópsia, perda do enxerto, óbito ou perda de seguimento) foi semelhante nos dois grupos (Tabela 2). A incidência de rejeição aguda clínica foi menor nos pacientes tratados com TAC, mas não entre pacientes tratados com TAC-MR quando comparados e observados entre pacientes que receberam ciclosporina. A incidência de rejeição aguda mais grave tratada com anticorpos policlonais foi menor nos pacientes tratados com TAC e TAC-MR, quando comparada e observada entre pacientes que receberam ciclosporina. A sobrevida dos enxertos e dos pacientes foi semelhante entre os grupos (Tabela 2).

A proporção de pacientes que completaram um ano de estudo recebendo a mesma medicação foi maior entre aqueles que receberam TAC ou TAC-MR comparado com ciclosporina (Tabela 3). Isso foi conseqüência tanto de maior segurança (descontinuação devido à reação adversa) como melhor eficácia (mudança após episódio de rejeição). O perfil geral de segurança do TAC-MR foi semelhante ao do TAC, observando-se diferenças significativas nas incidências de reações adversas comparadas com a ciclosporina. Pacientes recebendo TAC-MR ou TAC apresentaram melhor função renal, menores concentrações de colesterol, porém maior incidência de intolerância à glicose e diabete melito após o transplante (Tabela 4). Com relação às reações adversas, pacientes recebendo TAC-MR ou TAC apresentaram tipicamente maior incidência de diarréia, alopécia, tremor e insônia e aqueles que receberam ciclosporina mais hipertricose, hirsutismo, edema periférico e hiperplasia gengival (Tabela 4). ${ }^{18}$

Estes estudos indicam que TAC-MR, através da administração de uma dose única diária apresenta eficácia e segurança muito semelhante à do TAC, permitindo a utilização da mesma estratégia de monitoramento terapêutico amplamente empregado em pacientes

Tabela 2. Parâmetros de eficácia.

\begin{tabular}{|c|c|c|c|}
\hline & $\begin{array}{l}\text { TAC-MR } \\
(n=214)\end{array}$ & $\begin{array}{c}\text { TAC } \\
(n=212)\end{array}$ & $\begin{array}{c}\text { CsA } \\
(n=212)\end{array}$ \\
\hline Falha de eficácia, n (\%) & $30(14,0)$ & $32(15,1)$ & $36(17,0)$ \\
\hline Óbito, n & 3 & 9 & 5 \\
\hline Perda do Enxerto, n & 5 & 9 & 4 \\
\hline Rejeição aguda comprovada por biópsia, n (\%) & $22(10,3)$ & $16(7,5)$ & $29(13,7)$ \\
\hline Perda de seguimento, $n$ & 3 & 4 & 1 \\
\hline Risco Relativo (RR) vs. CsA & 0,83 & 0,89 & \\
\hline Diferença na incidência (\% vs. CsA) & $-3,0$ & $-1,9$ & \\
\hline Rejeição aguda tratada clinicamente (\%) & 18,2 & $11,8^{\star \star}$ & 21,2 \\
\hline Rejeição aguda tratada com anticorpos (\%) & $3,7^{*}$ & $2,8^{*}$ & 8,5 \\
\hline Sobrevida do Paciente, (\%) & 98,6 & 95,7 & 97,6 \\
\hline Sobrevida do Enxerto, (\%) & 96,7 & 92,9 & 95,7 \\
\hline
\end{tabular}

Falha de tratamento: rejeição aguda comprovada por biópsia, óbito, perda do enxerto ou perda de seguimento. 
Tabela 3. Taxas de descontinuação da medicação de estudo após um ano de transplante.

\begin{tabular}{lccc}
\hline Parâmetros (\%) & $\begin{array}{c}\text { TAC-MR } \\
(\mathbf{n = 2 1 4 )}\end{array}$ & $\begin{array}{c}\text { TAC } \\
(\mathbf{n = 2 1 2})\end{array}$ & $\begin{array}{c}\text { CsA } \\
(\mathbf{n = 2 1 2})\end{array}$ \\
\hline $\begin{array}{l}\text { Completaram o estudo recebendo a } \\
\text { medicação de estudo randomizada. }\end{array}$ & $85,5^{*}$ & $84,4^{*}$ & 71,2 \\
$\begin{array}{l}\text { Descontinuação da medicação de } \\
\text { estudo devido a Eventos Adversos }\end{array}$ & $8,9^{* *}$ & 10,8 & 17,5 \\
$\begin{array}{l}\text { Descontinuação da medicação de } \\
\text { estudo devido a Perda do Enxerto }\end{array}$ & 0,9 & 1,4 & 0,5 \\
$\begin{array}{l}\text { Descontinuação da medicação de } \\
\text { estudo devido a Rejeição }\end{array}$ & $0,5^{*}$ & $0^{*}$ & 7,5 \\
$\begin{array}{l}\text { Conversão a } \\
\text { Outros*** }\end{array}$ & $4,7^{*}$ & $2,8^{*}$ & 18,4 \\
\hline
\end{tabular}

a Foi permitida a conversão de TAC-MR para TAC ou CSA, de TAC para CsA e de CSA para TAC

${ }^{*}<0,001 ;{ }^{* *}<0,01 ;{ }^{* * *}$ incluindo os não-aderentes, retiradas de consentimento e perdas de seguimento

que recebem TAC. Estudos futuros com um número maior de pacientes seguidos por mais tempo podem determinar a influência da simplificação posológica na adesão do paciente e se as diferenças na farmacocinética do TAC podem resultar em menor incidência de reações adversas, principalmente a nefrotoxicidade. A confirmação dessas premissas certamente terá influência positiva e decisiva na sobrevida do receptor de transplante renal.
Tabela 4. Principais parâmetros de segurança e reações adversas.

\begin{tabular}{lccc}
\hline Parâmetros de segurança & $\begin{array}{c}\text { TAC-MR } \\
(\mathrm{n}=214)\end{array}$ & $\begin{array}{c}\text { TAC } \\
(\mathrm{n}=212)\end{array}$ & $\begin{array}{c}\text { CsA } \\
(\mathrm{n}=212)\end{array}$ \\
\hline Creatinina (mg/dL) & $1,39^{*}$ & 1,42 & 1,48 \\
Clearance de Creatinina (mL/min) & $58,7^{*}$ & $57,7^{*}$ & 54,6 \\
Colesterol total (mg/dL) & $189^{*}$ & $182^{*}$ & 202 \\
LDL colesterol (mg/dL) & $102^{*}$ & $97^{*}$ & 113 \\
Triglicéridos (mg/dL) & 184 & 177 & 196 \\
Glicemia em jejum > 126 mg/dLa & 56.4 & $64.0^{*}$ & 52.6 \\
Uso de insulina > 30 dias & 5.5 & 6.0 & 2.6 \\
Uso de hipoglicemiantes orais & $14.1^{*}$ & $10.0^{*}$ & 3.3 \\
\hline Reações adversas & & & \\
\hline Diarréia & $45,3^{* * *}$ & $44,3^{* * *}$ & 25,5 \\
Alopecia & $6,5^{*}$ & $7,1^{*}$ & 1,9 \\
Tremor & $35,0^{* * *}$ & $34,4^{* *}$ & 19,8 \\
Insônia & 25,7 & $30,2^{*}$ & 21,2 \\
Diabetes Mellitus & $14,0^{*}$ & 11,3 & 6,6 \\
Hipertricose & $0^{* *}$ & $0^{*}$ & 3,3 \\
Hirsutismo & $0^{* * *}$ & $0^{\star * *}$ & 8,5 \\
Edema Periférico & $35,5^{*}$ & $34,9^{*}$ & 45,8 \\
Hiperplasia Gengival & $0,5^{* *}$ & $0^{* *}$ & 4,7 \\
Hiperlipidemia & $16,4^{*}$ & 17,5 & 24,5 \\
\hline
\end{tabular}

a pelo menos uma glicemia de jejum igual ou acima de $126 \mathrm{mg} / \mathrm{dL}$ durante o primeiro ano do transplante.

b proporção de pacientes recebendo hipoglicemiante oral ao final do primeiro ano de transplante.

${ }^{*}<0,05 ;{ }^{* *}<0,01 ;{ }^{* * *}<0,001 ;$ TAC-MR vs. CsA ou TAC vs. CsA.

\section{ABSTRACT}

TAC-MR (TAC-MR) is a new extended release tacrolimus formulation that allows a unique daily dose. In health volunteers and in kidney and liver transplant recipients, tacrolimus exposition (AUC - area under the plasma-concentration curve) and residual concentration were similar after TAC or TAC-MR administration. The switch of TAC to TAC-MR is effective and safe, using the same monitoring strategy and same therapeutic range. Therapeutic equivalence between TAC and TAC-MR was proved in a prospective, randomized open study that included 638 new kidney transplant recipients. The average of tacrolimus doses and residual blood concentration, the ethnical influence and the difficulty to reach the therapeutic range were similar between renal transplant recipients that received TAC and TAC-MR. Differences in patients proportion that presented failure in the efficacy (endpoint composed by acute rejection proven by biopsy, graft loss, death or discontinuation) and in the safety profile.

Results of these studies indicate that TAC-MR, administered in a unique dose presents efficacy and safety similar to TAC, allowing the same therapeutic monitoring strategy. Expanded access programs studies are necessary to evaluate the TAC-MR influence in patient adherence to treatment, as well as if the pharmacokinetics differences could result in significantly adverse effects reduction, factor with a recognized impact on the recipient subject survival.

Keywords: Tacrolimus, Kidney Transplantation, Immunosuppression. 


\section{REFERÊNCIAS}

1. First R, Fitzsimmons W. Modified release tacrolimus. Yonsei medical journal 2004;45(6):1127-31.

2. Webster A, Woodroffe R, taylor R, Chapman J, Craig J. Tacrolimus versus cyclosporin as primary immunosuppression for kidney transplant recipients: metaanalysis and meta- regression of randomized trial data. BMJ. 2005;331(7520):810.

3. Seron D, O'valle F, Moresco F, Goma M, Huesco M, Grinyó J, et al. Immunophenotype of infiltrating cells in protocol renal allograft biopsies from tacrolimus-versus cyclosporine-treated patients. Transplantation. 2007;83(5):649- 52.

4. Vincenti F, Jensik S, Filo R, Miller J, Pirsch J. A long term comparison of tacrolimus (FK506) and cyclosporine in kidney transplantation: evidence for improved allograft survival at five years. Transplantation. 2002;73(5):75- 782 .

5. Sung In P, Felipe CR, Pinheiro-Machado PG, Garcia R, Tedesco-Silva H, Medina-Pestana JO. Circadian and time-dependent variability in tacrolimus pharmacokinetics. Fundamental \& Clinical Pharmacology. 2007;21:191-7.

6. Brochura do investigador, FK506E (MR4) Tacrolimus INN. Astellas, 2007.

7. Scott LJ, McKeage K, Keam S, Plosker G. Tacrolimus A further update of its use in the management of organ transplantation. Drugs. 2003;63(12):1247-97.

8. Cross S, Perry C. Tacrolimus Once-Daily Formulation: In the Prophylaxis of Transplant Rejection in Renal or Liver Allograft Recipients. Drugs. 2007;67(13):1931-43.

9. Wente M, Sauer P, Mehrabi A, Weitz J, Buchler M, Schmidt J, et al. Review of the clinical experience with a modified release form of tacrolimus [FK506E (MR4)] in transplantation. Clinical Transplantation. 2006;20(17):80-4.

10. Alloway R, Steinberg S, Khalil K, Gourishankar S, Miller J, Norman D, et al. Conversion of stable kidney transplant recipients from twice daily Prograf- based regimen to a once daily modified release tacrolimus-based regimen. Transplantation Proceedings. 2005;37:867-70.
11. Florman S, Alloway R, Kalayoglu M, Lake K, Bak T, Klein A, et al. Conversion of stable liver transplant recipients from a twice-daily Prograf-based regimen to a once-daily modified release tacrolimus-based regimen. Transplant Procceedings. 2005;37(2):1211-3.

12. Heffron T, Pescovitz M, Florman S, Kalayoglu M, Emre S, Smallwood G, et al. Once-daily tacrolimus extended- release formulation: 1-year post- conversion in stable pediatric liver transplant recipients. American Journal of Transplantation. 2007;7:1609-15.

13. Alloway R, for the tacrolimus modified release kidney transplant conversion study group. A two years follow up study of stable kidney transplant recipients converted from twice daily Prograf to once daily modified release tacrolimus. American Journal Transplant. Abstract \#752, 2006.

14. Florman $\mathrm{S}$, for the tacrolimus modified release liver transplant conversion study group. A two year follow up study of stable liver transplant recipients converted from twice daily Prograf to once daily modified release tacrolimus. American Journal Transplant. Abstract \#1936, 2006.

15. Heffron T. for the modified release pediatric liver concersion study group. American Journal Transplant. Abstract \#801, 2006.

16. Undre A. For the Tacrolimus Modified Release Kidney Study Group. Use of once a daily modified release tacrolimus regimen in de novo kidney transplant recipients. American Journal Transplant. 5:190, 2005. Abstract \# 132.

17. Undre A. For the Tacrolimus Modified Release Kidney Study Group. Use of once a daily modified release tacrolimus regimen in de novo liver transplant recipients. American Journal Transplant 5:374, 2005. Abstract \# 859.

18. Tedesco HT, Yang H, Abouljoud M, Kuo P, Wisemandle K, Bhattacharya P, Dhadda S, Holman J, Fitzsimmons W, et al. One-Year results with extended release tacrolimus/ MMF, tacrolimus/ MMF and cyclosporine/ MMF in de novo kidney transplant recipients. American Journal of Transplantation. 2007;7:1-14. 